\title{
Isolation of Organism and its Drug Sensitivity Pattern in Patients with Urinary Tract Infection at Kathmandu Model Hospital
}

\section{Joshi RD ${ }^{1}$, Khadka S ${ }^{1}$ Joshi DM¹, Dahal A ${ }^{1}$,Shrestha B ${ }^{2}$, Dangal G ${ }^{3}$}

${ }^{1}$ Department of Medicine, Kathmandu Model Hospital, Kathmandu, Nepal, ${ }^{2}$ Department of Microbiology, Kathmandu Model Hospital, Kathmandu, Nepal, ${ }^{3}$ Department of Obstetrics and Gynecology, Kathmandu Model Hospital, Kathmandu, Nepal.

Received: 14-May-2018; Accepted: 31-May-2018

Aims: Lack of compliance and unjustified antibiotic prescriptions has resulted in increasing bacterial resistance and is proving as a major challenge in the management of infections. Knowing the commonly isolated uropathogens and their antimicrobial susceptibility is beneficial in planning treatment protocols.

\begin{abstract}
Methods: A retrospective review of records of patients with urinary tract infection from January 2012 to December 2016 was conducted at Kathmandu Model Hospital. All patients who were diagnosed as having culture positive urinary tract infection in medical ward of Kathmandu Model Hospital during 5 years period were analyzed for demographic data, prevalence of organism and antibiotic susceptibility patterns.

Results: A total of 315 samples were culture positive. The majority of bugs were gram negative E.coli (48.57\%) followed by multi-drug resistant E.coli (28.89\%). The other major pathogens were E. faecalis $(6.03 \%)$, S. epidermidis $(4.44 \%), K$. pneumoniae $(4.13 \%)$, respectively. The highest level of sensitivity in first line antibiotics was seen in nitrofurantoin (84.9\%), whereas least sensitivity was shown by amoxicillin/clavulanic acid (21\%). Similarly, in second line antibiotics, highest sensitivity was seen in tetracycline $(100 \%)$, imipenem $(91.9 \%)$ and least to meropenem $(49.2 \%)$. Ceftazidime is mostly $(93.3 \%)$ resistant antibiotics among uropathogens. All the third line antibiotics such as polymyxin B, tigecycline and colistin were $100 \%$ sensitive to all our isolates.
\end{abstract}

Conclusions: Nitrofurantoin may be an appropriate choice for initial empirical therapy of urinary tract infection. Similarly, the multi-drug resistant E.coli is increasing but it can be tailored if antibiotics are used appropriately on the basis of susceptibility data.

Keywords: Colistin Sulphate; multi-drug resistant E.coli; Nitrofurantion

http://dx.doi.org/10.3126/njog.v13i1.21618

\section{INTRODUCTION}

Urinary tract infection (UTI) presents with lower abdominal pain, increase in urinary frequency, dysuria, low backache and fever, including acute and chronic pyelonephritis, cystitis, urethritis, epididymitis and prostatitis. It is one of the commonest infections in clinical practice and the second most common infectious presentation in community. Worldwide, there is an estimated 150 million UTIs per year. ${ }^{1,2}$ Lack of compliance and unjustified antibiotic prescriptions has resulted in increasing bacterial resistance and is proving as a major challenge in the management of this infection. ${ }^{3}$ Knowing the common isolated uropathogens and their

\section{CORRESPONDENCE}

Dr.Sachin Khadka,

Department of Medicine, Kathmandu Model Hospital,

Exhibition Road, Kathmandu, Nepal.

Email:sachinkhadka18@hotmail.com,

Phone: +9779840051772 antimicrobial susceptibility is beneficial in planning treatment protocols. Therefore, this study aimed to determine the bacteriological profile and antibiotic sensitivity patterns in UTI cases in our hospital over past five years.

\section{METHODS}

A retrospective review of records of patients with UTI from January 2012 to December 2016 was conducted at Kathmandu Model Hospital. All patients who were diagnosed as having culture positive UTI in medical ward of Kathmandu Model Hospital during 5 years period were analyzed for demographic data, prevalence of organism and antibiotic susceptibility patterns. Only the patients with urine cultures yielding significant growth of pathogens from a freshly voided midstream urine specimen were included in the study. Any patient records with incomplete information were excluded from this study. An antibiotic susceptibility pattern was further confirmed from the 
laboratory records. Antimicrobial susceptibility of the isolates was tested by the disc diffusion method. Due necessary permission from the concerned departments and Institutional Review Committee (IRC) of phectNEPAL/ Kathmandu Model Hospital were obtained. Data were entered and analyzed using Excel and SPSS version 21. Data has been summarized using percentage, graph, bar diagram, and tables.

\section{RESULTS}

Table 1.Demographic and clinical variables of Urinary tract infection ( $\mathrm{n}=315)$.

\begin{tabular}{lcc}
\hline Characteristics & \multicolumn{2}{c}{ Total (n)=315 } \\
& Number & Percentage (\%) \\
\hline Age & 20 & 6.3 \\
$\leq 20$ & 62 & 19.7 \\
\hline 21 to 40 & 79 & 25.1 \\
\hline 41 to 60 & 114 & 36.2 \\
\hline 61 to 80 & 40 & 12.7 \\
$\geq 81$ & & \\
\hline History of UTI & 55 & 17.5 \\
Yes & 260 & 82.5 \\
\hline No & & \\
\hline
\end{tabular}

Risk Factors (Diabetes, renal calculus, history of cauterization, VUR)

\begin{tabular}{lcc} 
Yes & 113 & 35.9 \\
\hline No & 202 & 64.1 \\
\hline Symptoms of UTI & & \\
\hline Yes & 174 & 55.2 \\
\hline No & 141 & 44.8 \\
\hline Pus cells in urine & & \\
Yes & 222 & 70.5 \\
\hline No & 93 & 29.5 \\
\hline
\end{tabular}

The mean age of study population is 56.1 years with a standard deviation of 21.19 (age distribution was 15 to 94). The data illustrates that the age group $61-80$ years is highly vulnerable (over one third) to UTI followed by 41-60 years group (a fourth) with the least vulnerable (marginally over a twentieth) being the population under 20 years of age. Majority $(82.5 \%)$ of patients did not have any history of UTI, lesser patients $(35.9 \%)$ presented the risk factors and almost similar number $(55.2 \%)$ of patients presented clinical symptoms. Lastly, while analyzing the presence of pus cells in the urine of culture positive patients, majority $(70.5 \%)$ showed positive result. [Table-1]
Table 2: Organisms causing urinary tract infection $(\mathrm{n}=315)$.

\begin{tabular}{lll}
\hline Bacteria isolated & Number (n) & Percent (\%) \\
\hline E. coli & 153 & $48.57 \%$ \\
MDR E. coli & 91 & $28.89 \%$ \\
E. faecalis & 19 & $6.03 \%$ \\
S. epidermidis & 14 & $4.44 \%$ \\
K. pneumoniae & 13 & $4.13 \%$ \\
Enterobacter Spp & 6 & $1.9 \%$ \\
S. saprophyticus & 3 & $1.0 \%$ \\
C. freundii & 3 & $1.0 \%$ \\
P. vulgaris & 3 & $1.0 \%$ \\
P. mirabilis & 2 & $.6 \%$ \\
Acinetobacter spp & 2 & $.6 \%$ \\
K. oxytoca & 2 & $.6 \%$ \\
P. aeruginosa & 2 & $.6 \%$ \\
C. koseri & 1 & $.3 \%$ \\
Coagulase Negative & 1 & $.3 \%$ \\
Staphylococci & & $100 \%$ \\
\hline Total & 315 & \\
\hline
\end{tabular}

The majority of bugs were gram negative aerobic rods. Among the gram negative rods, E.coli was most frequently UTI causing uropathogens, which accounted for $48.57 \%$ followed by multi-drug resistant E.Coli (28.89\%). The other major pathogens were E. faecalis $(6.03 \%)$, S. epidermidis $(4.44 \%), K$. pneumoniae $(4.13 \%)$, respectively. The prevalence of other uropathogens were almost similar in proportion. [Table-2]

Table 3 illustrates the sensitivity patterns of uropathogens to the first line, second line and third line antibiotics. The highest level of sensitivity in the first line antibiotics was seen in nitrofurantoin $(84.9 \%)$, gentamycin $(72.9 \%)$, levofloxacin $(54.5 \%)$, cotrimoxazole $(44.1 \%)$, norfloxacin (42.8\%), ofloxacin (42.2\%), and ciprofloxacin $(41.8 \%)$, whereas least sensitivity was shown by amoxicillin/clavulanic acid (21\%), amoxicillin (23.7\%), ceftriaxone (29.7\%), cefixime (37.4\%) and cefotaxime $(39.7 \%)$, respectively.

Similarly, the second line antibiotics also showed the mixed sensitivity patterns with highest sensitivity in tetracycline (100\%), imipenem (91.9\%), vancomycin (75\%) and piperacillin/tazobactam (74.4\%). Unfortunately, other carbapenen, mainly meropenem was sensitive in only $49.2 \%$ patients. Contrary to this, ceftazidime is mostly resistant antibiotics among uropathogens with $93.3 \%$ resistance. Fortunately, all the third line antibiotics polymyxin B, tigecycline and colistin were $100 \%$ sensitive to all our isolates. 
Table 3: Overall sensitivity of uropathogens ( $\mathrm{n}=315)$

\begin{tabular}{|c|c|c|c|c|}
\hline Antibiotics & Number (n) & Sensitivity (\%) & Resistance (\%) & Intermediate (\%) \\
\hline \multicolumn{5}{|l|}{ 1st Line } \\
\hline Amoxicillin & 152 & $36(23.7 \%)$ & $115(75.7 \%)$ & $1(0.6 \%)$ \\
\hline Amoxicillin/Clavulanic Acid & 38 & $8(21 \%)$ & $29(76.3 \%)$ & $1(2.6 \%)$ \\
\hline Cefixime & 278 & $104(37.4 \%)$ & $168(60.4 \%)$ & $6(2.2 \%)$ \\
\hline Cefotaxime & 295 & $117(39.7 \%)$ & $175(59.3 \%)$ & $3(1 \%)$ \\
\hline Ceftriaxone & 229 & $68(29.7 \%)$ & $159(69.4 \%)$ & $2(0.9 \%)$ \\
\hline Ciprofloxacin & 306 & $128(41.8 \%)$ & $167(54.6 \%)$ & $11(3.9 \%)$ \\
\hline Norfloxacin & 306 & $131(42.8 \%)$ & $164(53.6 \%)$ & $11(3.6 \%)$ \\
\hline Ofloxacin & 282 & $119(42.2 \%)$ & $155(55 \%)$ & $8(2.8 \%)$ \\
\hline Nitrofurantoin & 312 & $265(84.9 \%)$ & $39(12.5 \%)$ & $8(2.6 \%)$ \\
\hline Cotrimoxazole & 299 & $132(44.1 \%)$ & $165(55.2 \%)$ & $2(0.7 \%)$ \\
\hline Gentamycin & 214 & $156(72.9 \%)$ & $52(24.3 \%)$ & $6(2.8 \%)$ \\
\hline Levofloxacin & 145 & $79(54.5 \%)$ & $50(34.5 \%)$ & $16(11 \%)$ \\
\hline \multicolumn{5}{|l|}{$2^{\text {nd }}$ line } \\
\hline Amikacin & 180 & $117(37.1 \%)$ & $6(5.1 \%)$ & $2(.6 \%)$ \\
\hline Cefoperazone/Salbactam & 110 & $54(49 \%)$ & $50(45.5 \%)$ & $6(5.5 \%)$ \\
\hline Ceftazidime & 132 & $5(3.8 \%)$ & $124(93.9 \%)$ & $3(2.3 \%)$ \\
\hline Doxycycline & 46 & $24(52.2 \%)$ & $20(43.5 \%)$ & (4).30\% \\
\hline Imipenem & 99 & $91(91.9 \%)$ & $8(8.1 \%)$ & - \\
\hline Meropenem & 130 & $64(49.2 \%)$ & $60(52.2 \%)$ & $6(4.8 \%)$ \\
\hline Piperacillin/Tazobactam & 115 & $81(74.4 \%)$ & $30(26 \%)$ & $4(3.5 \%)$ \\
\hline Tetracycline & 3 & $3(100 \%)$ & - & - \\
\hline Vancomycin & 8 & $6(75 \%)$ & $2(25 \%)$ & - \\
\hline \multicolumn{5}{|l|}{$3^{\text {rd }}$ Line } \\
\hline Polymyxin B & 5 & $5(100 \%)$ & - & - \\
\hline Tigecycline & 9 & $9(100 \%)$ & - & - \\
\hline Colistin & 10 & $10(100 \%)$ & - & - \\
\hline
\end{tabular}

\section{DISCUSSION}

Urinary tract infections are the most frequent bacterial infection in women. In a study done in a rural area of India where the prevalence of UTI in female was $78.8 \%{ }^{4}$ The close proximity of the female urethral meatus to anus, short urethra, altered vaginal biota and sexual intercourse influence the higher prevalence of UTI in female. ${ }^{5}$ In addition, elderly populations have increased vulnerability towards UTI for various associated risk factors such as age-associated altered immunity, increased comorbid conditions and exposure to nosocomial pathogens. ${ }^{6}$ Our study showed almost three-fourth of the UTI patients were over 40 years, indicating the higher prevalence of UTI in elderly people. A similar finding was demonstrated by the study done by Prakash and Saxena where the highest susceptible age group of patient to UTI was over 48 year with $63.51 \%$ prevalence. ${ }^{7}$

In our study, majority $(82.5 \%)$ of the patients did not have any history of UTI which is similar to the study done by Derese et al where $73.1 \%$ patients had no history of UTI. ${ }^{8}$ Similarly, we observed predisposing factors for UTI were present in $35.9 \%$ patient contrast to $64.1 \%$ with no risk factor. In this study, condition like diabetes, renal calculus, history of cauterization, VUR are considered as predisposing factor. Overall, these entire predisposing factors play role in causing UTI but the association with UTI is not very strong as shown in study done in Nepal by Subedi et $\mathrm{al}^{9}$ and in study done by Holmgrem. ${ }^{10}$ Over viewing the clinical symptoms, almost similar number of patient had clinical symptom (55.2 vs. $44.8 \%$ ). Previous study also showed clinical symptoms were present in $47.3 \%$ which is nearly similar to our study. ${ }^{8}$ When analyzing the presence of pus cells in culture growth patients, $70.5 \%$ patients showed positive result. In this study, pus cells more than $6 / \mathrm{HPF}$ is considered positive. ${ }^{11} \mathrm{~A}$ cross sectional study from Bangladesh showed out of 100 urinary samples having pus cells $>5 / \mathrm{HPF}, 93.3 \%$ culture positive patients showed significant pyuria. ${ }^{12}$ Increasing resistance in bacterial pathogens is of world-wide concern. In this study, the majority of 
bugs were gram negative aerobic rods. Among the gram negative rods, E.coli was most frequently UTI causing uropathogens, which accounted for $48.57 \%$. Frequency of pathogens causing urinary tract infection in a tertiary care hospital in Western Nepal was studied, where the most common pathogens isolated were E. coli $(59.4 \%) .{ }^{13}$ Similarly, the study done in india showed E. coli was found positive in $61.02 \%$ samples. The second frequently occurred organism in our study is multi-drug resistant E.coli (28.89\%). In most of the study done in Nepal, other organisms like E. faecalis, S. epidermidis, K. pneumoniae are the second most causatives of UTI, ${ }^{14}$ but in our study MDR E. coli is the second most frequently occuring organism which is similar to the study done in Thailand where MDR E. coli is higher. ${ }^{15}$ The other major pathogens were E. faecalis $(6.03 \%)$, S. epidermidis (4.44\%), and K. Pneumoniae (4.13\%), respectively. Antibiotic susceptibility pattern of urinary isolates from Manipur showed Klebsiella species (14.4\%) is the second most common after E. coli. ${ }^{16}$ In a prospective study undertaken over a 14-month period in Iran, E. coli was the most common etiological agent of UTI (74.6\%), followed by Klebsiella spp (11.7\%), S. saprophyticus (6.4\%), and P. aeruginosa $(2.2 \%) .{ }^{17}$ In another study, Klebsiella species caused urinary tract infection in maximum number of cases $(124,37.35 \%)$ followed by $E$. coli $(114,34.4 \%) .{ }^{18}$ The prevalence of antimicrobial resistance in both out and hospital patients with UTI is increasing and can vary according to geographical and regional location, but in overall, E. coli is causing UTI in most of the people worldwide.

Regarding the sensitivity patterns of uropathogens to first line antibiotics, the highest level of sensitivity was seen with nitrofurantion $(84.9 \%)$, gentamycin $(72.9 \%)$, levofloxacin $(54.5 \%)$, cotrimoxazole (44.1\%), norfloxacin (42.8\%), ofloxacin (42.2\%), and ciprofloxacin $(41.8 \%)$, whereas the least sensitivity was shown by amoxicillin/clavulanic acid (21\%), amoxicillin 36 (23.7\%), ceftriaxone (29.7\%), cefixime (37.4\%) and cefotaxime (39.7\%). Considering antibiotic sensitivity, our results were different from a year-long study conducted in Shankarapur Hospital in Kathmandu valley in 2015 where the sensitivity rates of nitrofurantion (59.4\%), gentamycin $(56.4 \%)$ were lesser than our findings. Whereas similar comparable sensitivity was seen with cotrimoxazole (47.3\%), ofloxacin (50\%), ciprofloxacin $(49.7 \%)$, and cefixime $(48.5 \%)$. In addition, a higher antibiotic sensitivity to ceftriaxone (73.3\%\%) and amoxicillin/clavulanic acid (60.6\%\%) was seen which is contrary with our finding. ${ }^{9}$ Other study done at Bangladesh showed cotrimoxazole and amoxicillin are virtually useless against uropathogens as they were effective against $31.81 \%$ and $11.81 \%$ of all isolated organisms respectively. This study also showed gentamycin is sensitive in $74.54 \%$ which is almost similar to our study but amikacin sensitivity is much higher than our study $(86.3 \%) .{ }^{19}$ Reduced susceptibility to amoxicillin in our study may indicate patients presenting to primary care which may have less severe symptoms and likely to present earlier or may reflect changes in antibiotic susceptibilities due to physicians' prescribing behavior. ${ }^{20}$

Similarly, second line antibiotics also showed the mixed sensitivity patterns with highest sensitivity with tetracycline (100\%), imipenem $(91.9 \%)$, vancomycin $(75 \%)$ and piperacillin/tazobactam (74.4\%). The study done by Subedi et al showed similar sensitivity to tazobactam (73.3\%). ${ }^{9}$ Similarly, study from Bangladesh showed imipenem and meropenem is $98.18 \%$ sensitive to uropathogens whereas our study shows similar result with imipenem (91.9\%) but, unfortunately meropenem was sensitive in only $49.2 \%$ patients. According to our results, ceftazidime was $3.6 \%$ sensitive which differ from a Pakistani study done by Tabish and Iqbal where ceftazidime sensitivity was $46.66 \%{ }^{20}$

In our study, overall the third line antibiotics polymyxin $\mathrm{B}$, tigecycline and colistin were $100 \%$ sensitive to all our isolates. Identical results are reported in a study from USA where tigecycline were found to be most efficacious. ${ }^{21}$

\section{CONCLUSIONS}

In conclusion, nitrofurantion may be an appropriate choice for initial empirical therapy of UTI though the uropathogens showed high levels of resistance to multiple urinary antimicrobial agents. Similarly, the multi-drug resistant E.coli is increasing but it can be tailored if antibiotics are used appropriately on the basis of susceptibility data. The third line antibiotics polymyxin B, tigecycline and colistin could be used only if needed to preserve it for future for MDR uropathogens.

\section{ACKNOWLEDGEMENTS}

The authors would also like to thank all participants in this study and the laboratory staffs for their help during the study. 


\section{REFERENCES}

1. Gales AC, Sader HS, Jones RN. The SENTRY Participants Group (Latin America). Urinary tract infection trends in Latin American hospitals: Report from the SENTRY antimicrobial surveillance program (1997-2000). Diagn Microbiol Infect Dis 2002; 44:289-9

2. Mirzarazi M, Rezatofghi ES, Pourmahdi M, Mohajeri RM. Antibiotic resistance of isolated gram negative bacteria from urinary tract infections (UTIs) in Isfahan. Jundishapur J Microbiol. 2013; 6(8):e6883. doi:10.5812/jjm.6883

3. Arslan B, Kozacioglu Z, Ergin OY, Bozkurt IH. Degirmenci T, Yonguc T, Gunlusoy B. Pathogen bacteria of the urinary tract isolated from urine cultures and their susceptibility. Erciyes Med J. 2014; 36:29-34

4. Dash M, Padhi S, Mohanty I, Panda P, Parida B. Antimicrobial resistance in pathogens causing urinary tract infections in a rural community of Odisha, India. J Fam Comm Med. 2013; 20(1):20-6.

5. Minardi D, d'Anzeo G, Cantoro D, Conti A, Muzzonigro G. Urinary tract infections in women: etiology and treatment options. Int J Gen Med. 2011;4: 333-43.

6. Rowe TA, Juthani-Mehta M. Urinary tract infection in older adults. Aging Health. 2013;9(5): 10.2217/ahe.13.38.

7. Prakash D, Saxena RS. Distribution and antimicrobial susceptibility pattern of bacterial pathogens causing urinary Tiract infection in urban community of Meerut City, India. ISRN microbiology. 2013. 749629. 10.1155/2013/749629.

8. Derese B, Kedir H, Teklemariam Z, Weldegebreal F, Balakrishnan S. Bacterial profile of urinary tract infection and antimicrobial susceptibility pattern among pregnant women attending at Antenatal Clinic in Dil Chora Referral Hospital, Dire Dawa, Eastern Ethiopia. The Clin Risk Manag. 2016;12: 251 - 60. http://doi.org/10.2147/TCRM.S99831

9. Subedi N, Pudasaini S. Bacteriological profile and antibiotic sensitivity pattern in patients with urinary tract infection. Journal of Pathology of Nepal. 2017;7(1): 1066-1069. doi:http://dx.doi.org/10.3126/jpn.v7i1.16910.

10. Holmgren K. Urinary calculi and urinary tract infection. A clinical and microbiological study. Scand J Urol Nephrol Suppl. 1986; 98:1-71.

11. Bates BN. Interpretation of urinalysis and urine culture for UTI treatment. US Pharm. 2013;38(11):65-8.
12. Parvin US, Hossain MA, Musa AK, Mahamud C, Islam MT, Haque N, et al. Pattern of aerobic bacteria with antimicrobial susceptibility causing community acquired urinary tract infection. Mymensingh Med J. 2009; 18(2):148-53.

13. Das RN, Chandrasekhar TS, Joshi HS, Gurung M, Shrestha $\mathrm{N}$, Shivananda PG. Frequency and susceptibility profile of pathogens causing urinary tract infections at a tertiary care hospital in western Nepal. Singapore Med J. 2006; 47(4):2815 .

14. Shrestha P, Malla S, Basnyat S, Dumre S, Upadhyay B, Lamichhane $\mathrm{S}$. Antimicrobial resistance pattern of bacterial isolates causing urinary tract infection. J Nepal Health Res Counc. 2007; 5(2): 49-54. DOI: 10.3126/jnhrc.v5i2.2471

15. Khawcharoenporn $\mathrm{T}$, Vasoo $\mathrm{S}$, Singh $\mathrm{K}$. Urinary tract infections due to multidrug-resistant Enterobacteriaceae: prevalence and risk factors in a Chicago Emergency Department. Emerg Med Int. 2013; 2013:7.

16. Yengkokpam C, Ingudam D, Yengkokpam IS, Jha BK Antibiotic susceptibility pattern of urinary isolates in Imphal (Manipur) India. Nepal Med Coll J. 2007; 9(3):170-2.

17. Farjinia S, Alikhani MY, Ghotaslou R, Naghili B, Nakhlband A. Causative agents and antimicrobial susceptibilities of urinary tract infections in the northwest of Iran. Int J Infect Dis 2009;13(2):140-4.

18. Bajaj JK, Karyakarte RP, Kulkarni JD, Deshmukh AB Changing aetiology of urinary tract infections and emergence of drug resistance as a major problem. J Commun Dis. 1999; 31(3):181-4

19. Tabish Humayun, Abida Iqbal. The Culture and Sensitivity Pattern of Urinary Tract Infections in Females of Reproductive Age Group. Ann Pak Inst Med Sci. 2012; 8(1): 19-22.

20. Wong CKM, Kung K, Au-Doung PLW, Ip M, Lee N, Fung A, et al. Correction: Antibiotic resistance rates and physician antibiotic prescription patterns of uncomplicated urinary tract infections in southern Chinese primary care. PLoS ONE. 2018;13(2): e0192466. https://doi.org/10.1371/journal. pone. 0192466

21. Kelesidis T, Karageorgopoulos DE, Kelesidis I, Falagas ME. Tigecycline for the treatment of multidrug-resistant Enterobacteriaceae: a systematic review of the evidence from microbiological and clinical studies. J Antimicrob Chemother 2008; 62(5):895-904. doi: 10.1093/jac/dkn311. 